\title{
Polarization modulation laser scanning microscopy: A powerful tool to image molecular orientation and order
}

\author{
Vinay K. Gupta and Julia A. Kornfield \\ Division of Chemistry and Chemical Engineering, 210-41, California Institute of Technology, Pasadena, \\ California 91125
}

(Received 18 April 1994; accepted for publication 17 May 1994)

\begin{abstract}
To image the orientational order in a broad class of biological and manufactured materials, a new microscope has been developed that integrates laser scanning microscopy with polarization modulation polarimetry. Polarimetry allows quantitative characterization of the molecular orientation and the degree of order through characterization of optical anisotropy. Combined with laser scanning microscopy, it is used here to image the anisotropy with high spatial resolution, sensitivity, and speed. The design of the microscope is presented; and the vast improvement in sensitivity achieved using PM-LSM over conventional polarization microscopy is illustrated by imaging the linear dichroism of ultrathin Langmuir-Blodgett polymer films. PM-LSM allows imaging of the magnitude and orientation of linear dichroism in films as thin as three molecular layers $(\sim 66 \AA)$ at high resolution by rastering a diffraction limited spot of laser light across the sample. The rate of image acquisition is over 2000 pixels/s, two to three orders of magnitude faster than the previous methods of imaging optical anisotropy.
\end{abstract}

\section{INTRODUCTION}

Images of the optical anisotropy of oriented materials are measured to investigate a wide range of phenomena, including the development of cell structure, the phase transitions of liquid crystals, and the stress distribution in solids under load. ${ }^{1-5}$ Two types of optical anisotropy are observed: dichroism, the anisotropic attenuation, and birefringence, the anisotropic retardation of polarized light. Conventional methods of polarization imaging on a microscopic scale involve using optical microscopy with the sample between crossed polarizers, and provide only qualitative information. Moreover, the use of a broadband light source blurs the image. This blurring is worsened by the relatively poor extinction ratios of the film polarizers that are used. Even if a monochromatic source and ideal polarizers are used, the magnitude and orientation of birefringence or dichroism cannot be determined from the measured intensity image alone.

To construct a microscope that can rapidly and quantitatively record birefringence and dichroism images, we combine scanning optical microscopy and polarizationmodulation polarimetry. The availability of bright, collimated laser sources has resulted in the development of methods to scan a diffraction-limited spot over a microscopic area in order to increase the acquisition speed and resolution of images. ${ }^{6-8}$ Concurrent developments in polarimetry, ${ }^{9-11}$ the measurement of the polarization state of light, have taken advantage of laser sources in combination with highfrequency polarization-modulating optics to greatly improve the sensitivity and response time of measurements of the magnitude and orientation of birefringence and dichroism in a material.

The feasibility of polarization-modulation optical microscopy has been demonstrated by the construction of two prototype microscopes ${ }^{1-3,12}$ that use nearly collimated, monochromatic UV light and incorporate the necessary modulating optics and demodulating electronics. In one of the prototypes, full-field illumination is used and a diode array camera records the image. This design provides an image acquisition rate of about 30 pixels/s. In the second prototype, the sample is moved in a raster fashion with respect to a diffraction limited spot of light. This technique provides greater sensitivity and spatial resolution with about the same acquisition speed. Both prototypes, however, require that the image be acquired at two different orientations in order to quantitatively determine both the magnitude and orientation of the dichroism.

We have equipped a laser scanning microscope with polarization modulation of the incident beam and demodulation of the detected intensity, which allows simultaneous acquisition of both the magnitude and orientation of the birefringence or dichroism. By using a bright, monochromatic, collimated source, the laser scanning system intrinsically provides significantly greater sensitivity than previous techniques. This enables much higher speed image acquisition (over $2000 \mathrm{pixels} / \mathrm{s}$ ) and makes it possible to follow transient phenomena such as the physiological response of a cell or the defect dynamics of condensed systems, anong many others. We present here a complete description of the instrument and its operation. Images of ultrathin Langmuir-Blodgett films are acquired using polarization modulation laser scanning microscopy. Characterization of the molecular order, in a nondestructive manner, in LB films is of great significance due to their potential application in molecular electronic devices and display devices. We show that PM-LSM can provide spatially resolved information on molecular alignment in films as thin as three molecular layers.

\section{POLARIZATION MODULATION POLARIMETRY}

Polarimetry ${ }^{9}$ is the measurement of changes in polarization of light emanating from the sample to analyze the structural anisotropy of the system under study. The polarization of light is affected by the material's refractive index tensor 


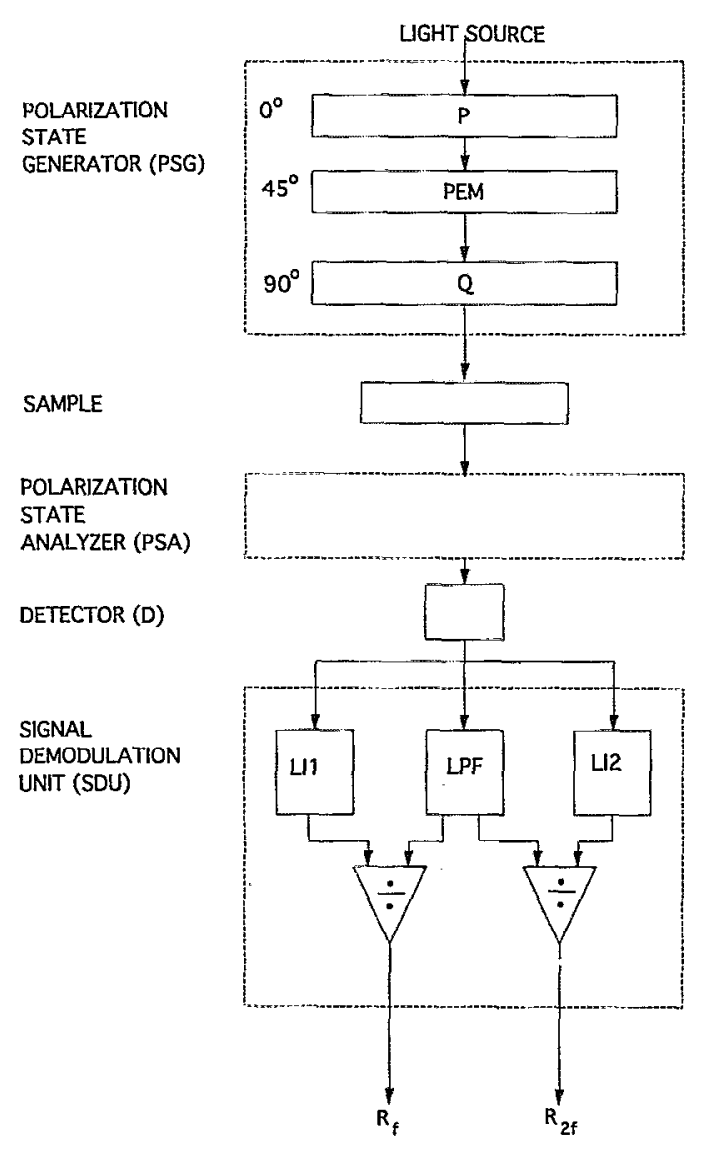

FIG. 1. Polarimetric arrangement for determination of linear dichroism or linear birefringence. The polarization state generator (PSG) consists of a linear polarizer (P), photoelastic modulator (PEM) of frequency $f$, and a quarter-wave plate (Q). The polarization state analyzer (PSA) is chosen as described in the text. Signal analysis is performed by a signal demodulation unit (SDU) consisting of two lock-in amplifiers, one at the fundamental $f$ (LI1) and the other second harmonic $2 f$ (LI2) of the modulation frequency, a low-pass filter (LPF) and two ratio circuits.

$\left(n_{i j}^{\prime}+i n_{i j}^{\prime \prime}\right)$ in two ways: it may be altered by anisotropic attenuation (dichroism, $\Delta n^{\prime \prime}$ ) and/or by anisotropic retardation (birefringence, $\Delta n^{\prime}$ ). In structurally anisotropic materials, the magnitude and orientation of these quantities can be related to the orientational correlation that exists in the material, in particular, the direction of the optical axis and the degree of order. Thus it becomes possible to quantify the orientation field in the sample by the pattern of birefringence or dichroism that is observed.

High sensitivity in the measurement of the optical anisotropies can be achieved by introducing a carrier frequency at which the polarization state of the light beam is modulated. ${ }^{10,11}$ As a result of polarization modulation, the need for rotating the polarizers or the sample is obviated. A rapid dynamic response is achieved, and the higher sensitivity makes it possible to measure birefringence or dichroism in extremely thin samples.

The modulation scheme we use (Fig. 1) employs a photoelastic modulator (PEM) that has a sinusoidal time-varying phase retardation $\left(\delta_{0} \sin f t\right)$. This arrangement produces a linearly polarized beam of constant intensity and an orientation that sweeps back and forth through a wide angle at a frequency $f$. As the polarization direction of the beam sweeps back and forth, it probes the orientation dependence of the properties of the sample. If the material is linearly dichroic, then the attenuation of beam rises and falls as its orientation sweeps back and forth. By analyzing the resulting modulation in intensity, we may determine the absorption anisotropy $(\nu)$ and its orientation angle $(\chi)$. In particular, if we demodulate the intensity into its steady part $\left(I_{d c}\right.$, from the low-pass filter, LPF) and its first and second harmonics $\left(I_{f}\right.$ and $I_{2 f}$, from two lock-in amplifiers, LI1 and LI2) (Fig. 1), the normalized harmonics can be used to determine $\nu$ and $\chi$ using the following relations: ${ }^{13}$

$$
\begin{aligned}
& R_{f} \equiv I_{f} / I_{d c}=\frac{2 J_{1}\left(\delta_{0}\right) \sin (2 \chi) \sinh (\nu)}{\cosh (\nu)-J_{0}\left(\delta_{0}\right) \cos (2 \chi) \sinh (\nu)}, \\
& R_{2 f} \equiv I_{2 f} / I_{d c}=\frac{2 J_{2}\left(\delta_{0}\right) \cos (2 \chi) \sinh (\nu)}{\cosh (\nu)-J_{0}\left(\delta_{0}\right) \cos (2 \chi) \sinh (\nu)},
\end{aligned}
$$

where $J_{0}, J_{1}$, and $J_{2}$ are calibration constants determined using samples of known optical anisotropy, and $\nu=2 \pi \Delta n^{\prime \prime} d / \lambda$ for an optical path length $d$, and wavelength of light $\lambda$.

For the case of a sample that is birefringent with little or no dichroism, only the phase of the transmitted beam is anisotropically affected by the sample. That is, as the orientation of the incoming polarization swings back and forth, the degree of ellipticity of polarization state of the emerging beam rises and falls. This modulation of the ellipticity of the polarization state may be translated into a measurable intensity variation using a circular polarizer as the analyzer (PSA in Fig. 1). In this case,

$$
\begin{aligned}
& R_{f}=\frac{-2 J_{1}\left(\delta_{0}\right) \cos (2 \chi) \sin (\mu)}{1-J_{0}\left(\delta_{0}\right) \sin (2 \chi) \sin (\mu)}, \\
& R_{2 f}=\frac{2 J_{2}\left(\delta_{0}\right) \sin (2 \chi) \sin (\mu)}{1-J_{0}\left(\delta_{0}\right) \sin (2 \chi) \sin (\mu)},
\end{aligned}
$$

where $\mu$ is proportional to the magnitude of birefringence $\left(\mu=2 \pi \Delta n^{\prime} d / \lambda\right)$, and $\chi$ its orientation. ${ }^{13}$

\section{POLARIZATION MODULATION LASER SCANNING MICROSCOPY}

Polarimetric techniques are typically used with a fixed beam, providing information that is spatially averaged over the illuminated spot. ${ }^{14-16}$ To obtain a spatially resolved image of the anisotropy, polarization-modulation polarimetry is combined with scanning optical microscopy. In scanning optical microscopy ${ }^{6-8}$ the object is not illuminated as a whole but is illuminated point by point, and the transmitted (or reflected or scattercd) light is measured using photodetectors. The image is built up pixel by pixel by scanning either the sample or the incident beam. Scanning microscopy and polarimetry have been recently combined in differential polarization imaging to probe the molecular organization in biological specimens. ${ }^{1-3,12}$ Images are constructed from the magnitude of linear and circular dichroism. The basic principle behind these microscopes can be extended to provide simultaneous determination of both the magnitude and the orientation angle of dichroism and birefringence. And vast 
improvements in sensitivity and speed can be achieved by using laser light and rastering the beam rather than the sample. . $^{3,12}$

To achieve these improvements, we have integrated a polarization-modulation optical arrangement in a Zeiss laser scanning microscope (LSM) ${ }^{7,8}$ As the laser beam rasters across the sample, the two lock-in signals from each pixel are normalized by the steady component of the intensity. The two ratios $R_{f}$ and $R_{2 f}$ are calculated at each pixel. We modify the electronics of the LSM to record these two signals. Using this information, we create an image of the orientation of the optical axis $\chi$ and degree of ordering, proportional to $\nu$ or $\mu$, from Eqs. (1) and (2) or (3) and (4), as appropriate for the sample. The construction and operation of this instrument are described in the next section.

\section{INSTRUMENT DESIGN AND OPERATION}

In the Zeiss laser scanning microscope a polarized laser beam is focussed to a diffraction-limited spot, which is rastered across the image very rapidly using a servo-controlled, feedback galvanometer system. A dual laser option provides laser light at wavelengths of 514 and $488 \mathrm{~nm}$ for an Argonion laser and at $543 \mathrm{~nm}$ for a green Helium-Neon laser. Electronic zoom over a range of 1:8 combined with appropriate lenses permits a field of view ranging from 6000 $\times 4000 \mu \mathrm{m}$ to $19 \times 14 \mu \mathrm{m}$. The Zeiss LSM also allows the simultaneous display of images constructed from two independently acquired signals. This feature is extremely valuable in our scheme of PM-LSM as we record the two ratios $R_{f}$ and $R_{2 f}$ simultaneously.

Our modifications of the LSM have been designed to facilitate changing between operation in the conventional laser-scanning mode and the new polarization-modulation laser-scanning mode. The modifications can be divided into two major categories.

(1) Optical modifications: A special polarization modulating unit has been fabricated that contains all three components of the polarization state generator of our optical train (PSG in Figs. 1 and 2). The analyzer components which include a collecting lens and circular polarizer (for birefringence images), go beneath the sample stage in the microscope (Fig. 2).

(2) Electronic modifications to record the normalized lock-in amplifier signals $R_{f}$ and $R_{2 f}$ that are needed to measure the magnitude and orientation of birefringence or dichroism. We have equipped the LSM with an external silicon photodiode that intercepts the laser beam (Fig. 2). The signal from the detector is demodulated by the lock-in amplifiers (Fig. 1). The lock-in outputs are normalized by the mean intensity $\left(I_{d c}\right)$ provided by a low-pass filter (Fig. 1) and then fed to the signal processing unit of the LSM via a relay switch (Fig. 2).

Here, we describe these optical and electronic modifications in more detail. The polarization modulating unit consists of a linear film polarizer, a Hinds International photoelastic modulator (PEM) oriented at $45^{\circ}$ with respect to the polarizer and a quarter-wave retarder oriented at $90^{\circ}$ with respect to the polarizer (Fig. 1). A half-wave plate can be used above the PSG module to rolate the polarization direc-

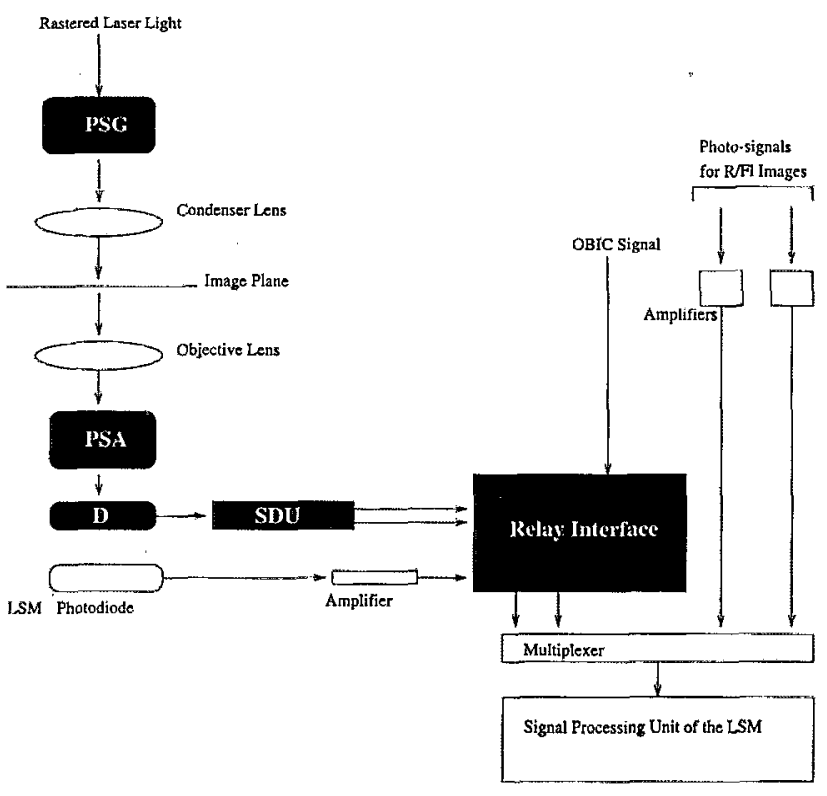

FIG. 2. Modifications of the laser scanning microscope. The polarization state generator (PSG), analyzer (PSA), and signal demodulation unit (SDU) are as described in Fig. 1.

tion of the laser light and bring it parallel to the linear polarizer ( $P$ in Fig. 1). The frequency of the modulator is $f=63$ $\mathrm{kHz}$ and the amplitude $\left(\delta_{0}\right)$ of its sinusoidal retardation set such that

$$
J_{0}\left(\delta_{0}\right)=0 \text {, }
$$

so that Eqs. (1) and (2) simplify to

$$
\begin{aligned}
& R_{f}=2 J_{1}\left(\delta_{0}\right) \sin (2 \chi) \tanh (\nu), \\
& R_{2 f}=2 J_{2}\left(\delta_{0}\right) \cos (2 \chi) \tanh (\nu),
\end{aligned}
$$

and Eqs. (3) and (4) simplify to

$$
\begin{aligned}
& R_{f}=-2 J_{1}\left(\delta_{0}\right) \cos (2 \chi) \sin (\mu), \\
& R_{2 f}=2 J_{2}\left(\delta_{0}\right) \sin (2 \chi) \sin (\mu) .
\end{aligned}
$$

After passing through the appropriate analyzing optics as described earlier, the laser light is intercepted by the externally provided Si photodiode (Universal Detector, 6pinDP) where the light intensity is converted to an analog voltage signal. The steady part of this signal, $I_{d c}$, is extracted using a Stanford Research SR-640 low-pass filter (LPF in Fig. 1). Synchronous demodulation is performed using two lock-ins: a Stanford Research SRS850 digital lock-in amplifier (LI1 in Fig. 1) and an Ithaco 3962A lock-in amplifier (LI2 in Fig. 1). The scanning rate is limited by the requirement that the dwell time per pixel be sufficiently large for the lock-ins to isolate the two harmonics. This can be achieved by keeping the dwell time per pixel about five times the time constant of the low-pass filter circuit in the lock-in. Here, we are limited by the minimum time constant of $100 \mu$ s for Ithaco 3962A; the SR 850 has a $10 \mu$ s minimum time constant. The first and second harmonics obtained from the two lock-ins are normalized by $I_{d c}$ using two ratiometer circuits and transmitted to the microscope signal processor via the relay interface and protection circuit. The relay switch toggles between the two 
regular internal LSM signals and the two external signals (Fig. 2). Using the Zeiss LSMs unique capability to record the two signals simultaneously, the dual grey-scale image is saved in the LSMs internal format. Image analysis is subsequently performed on a Sun workstation (Sparc2).

Calibration is performed using a linear polarizer and the images for $J_{1}$ and $J_{2}$ are recorded. Given these values of $J_{1}$ and $J_{2}$, the grey level values for each pixel in the image of a sample are converted into the magnitude $(\nu$ or $\mu$ ) and orientation $(\chi)$ values for that pixel. Using these, the whole image is reconstructed, pixel by pixel, for both the magnitude and orientation.

\section{RESULTS AND DISCUSSION}

To demonstrate the capability of this new tool and illustrate the vast improvement in sensitivity and speed over previous instruments, we image the molecular orientation and order in ultrathin Langmuir-Blodgett films of a "hairy-rod" polymer [Fig. 3(a)]. These macromolecules consist of asymmetrically alkoxy-substituted phthalocyanines linked together by a siloxane backbone [Tetra(methoxy)tetra(octyloxy)-phthalocyaninatopolysiloxane, PcPs) $].{ }^{17-19}$ The phthalocyanine-based rodlike polymers are extremely good candidates because the phthalocyanine molecule is a dye that is highly optically anisotropic. PcPs absorbs strongly under visible light and exhibits a maximum absorption anisotropy at wavelengths around $550 \mathrm{~nm}$. Polymeric arrangement of the phthalocyanine rings co-facially with siloxane linkages results in an absorption anisotropy with a principal axis along the rod. Wegner and co-workers have found $d^{17,20,21}$ that in deposited films of asymmetrically substituted phthalocyaninato-polysiloxane the rodlike molecules lie on average in an orientationally ordered state.

Thin films of different thicknesses are deposited on a microscope glass slide by dipping the slide to different lengths [Fig. 3(b)]. Dichroism images are recorded at the boundaries between the bands as shown by the small squares. All images discussed in this section are acquired in $512 \times 256$ pixel frames and are recorded at a rate of $64 \mathrm{~s} \mathrm{per}$ frame. In comparison, the earlier prototype differential polarization microscopes were limited to acquiring a single image in a minimum time of $33 \mathrm{~min}$, and consequently required over an hour to obtain a map of both absorption anisotropy and its orientation.

The dichroism field observed at the boundary between a region covered with 31 molecular layers $(\sim 682 \AA)$ and a region covered with 21 layers $(\sim 462 \AA)$ of PcPs is shown in Fig. 4(a). The absorption anisotropy $(\nu)$ has been color coded for enhanced viewing contrast from red to blue (as $\nu$ increases) as shown in the accompanying color scale. The dichroism fields at the 21/11 layer boundary, 11/5 boundary, and $5 / 3$ boundary are shown in Figs. 4(b), 4(e) and 4(f), respectively. The absorption anisotropy shows a monotonic decrease from 31 molecular layers to 3 layers as is evident from the color shading from one dichroism image to the other. The relative decrease in $\nu$ across a boundary is much larger when the decrease in the thickness across the boundary is greater. This is seen clearly by the higher color contrast that corresponds to a step of 10 layers $(\sim 220 \AA)$ in the the
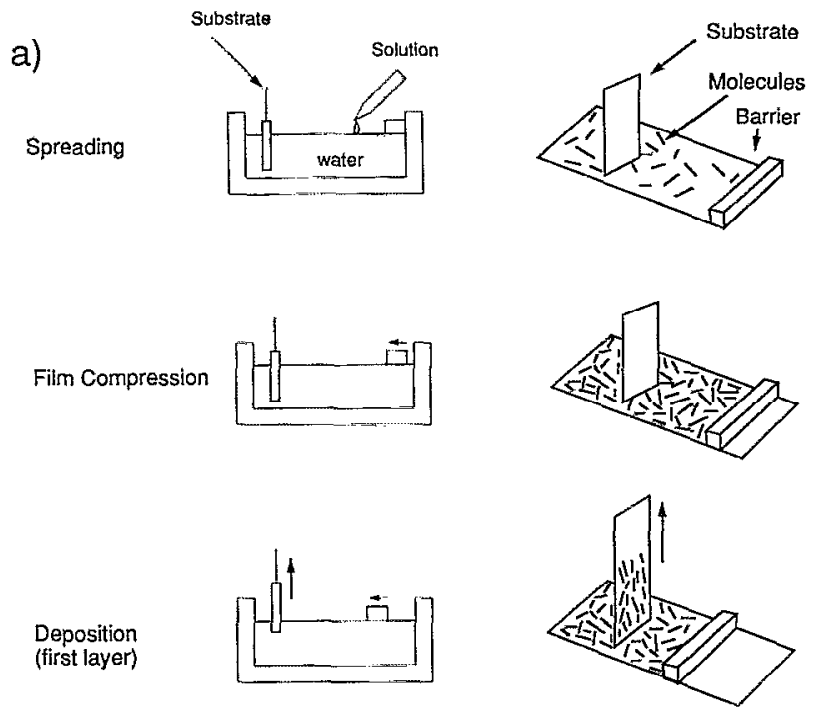

b)

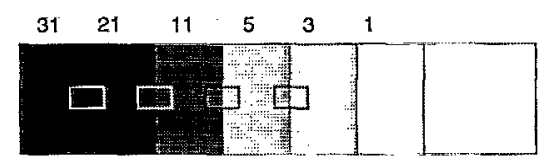

FIG. 3. (a) Preparation of a monomolecular layer on the air-water interface and transfer to a solid substrate. Subsequent layers are built upon the first layer by dipping of substrate through the interface, each dipping cycle depositing two additional layers. (b) Schematic diagram of the transferred layers of pthalocyaninato-polysiloxane (PcPs). The glass slide has bands with $1,3,5,11,21$, and $31 \mathrm{PcPs}_{\mathrm{S}}$ layers where each layer is approximately 22 $\AA$ in thickness. The square frames represent the approximate locations where linear dichroism images were acquired.

dichroism images in Figs. 4(a) and 4(b), compared to a lower contrast for a step of 6 layers [Fig, 4(e)] and one that is nearly negligible for a step of two molecular layers [Fig. 4(f)]. The decrease in $\nu$ in going from 31 layers to 3 layers and the steep drop at each boundary is seen easily in the graphs of the vertical line averages for each image, shown in Figs. 4(c), 4(d), 4(g), and 4(h) for the dichroism images Figs. 4(a), 4(b), 4(e), and 4(f), respectively.

Multiple layers are built up on the foundation of a monolayer on the glass substrate by withdrawing and dipping the slide up and down through the Langmuir monolayer, with each cycle depositing two layers [see Fig. 3(a)]. Ideally, the boundary between the two regions would be sharp, if the dipping process were carried out to precisely the same depth. However, we see that while the edge is fairly sharp at the $31 / 21$ layer boundary, there is a stair-step transition at the $21 / 11$ and $11 / 5$ boundaries [Figs. 4(b) and 4(e)], as also evident in the corresponding graphs of the line average [Figs. $4(\mathrm{c})$ and $4(\mathrm{~g})]$. This stair stepping is more pronounced in the $11 / 5$ layer boundary than in the $21 / 11$ layer boundary, and from the relative heights of the two steps in Fig. 4(g) we can surmise that the larger step corresponds to a decrease of 4 layers in thickness and that the next step to a decrease of 2 layers. Thus the narrow orange band, about $120 \mu \mathrm{m}$ wide, in Fig. 4(e) corresponds to a region with 7 molecular layers. Similarly, the narrow yellow band in Fig. 4(b) appears to be 
a)
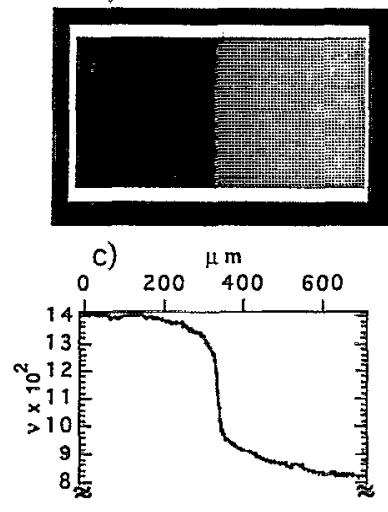

e)

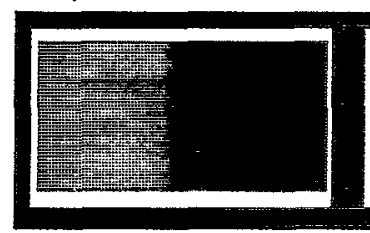

g) $\mu \mathrm{m}$

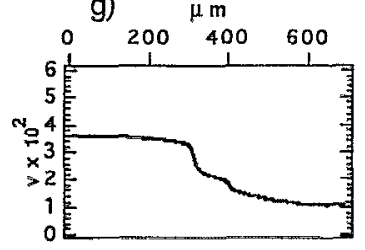

b)

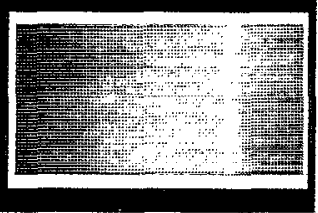

$0^{d)} 200 \quad \stackrel{\mu m}{400} 600$

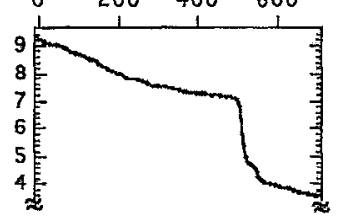

f)

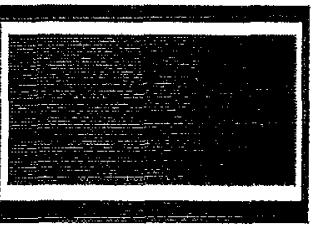

h) $\mu \mathrm{m}$

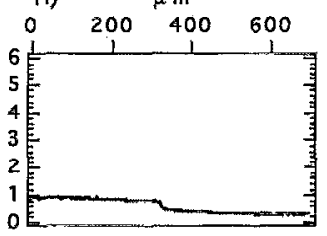

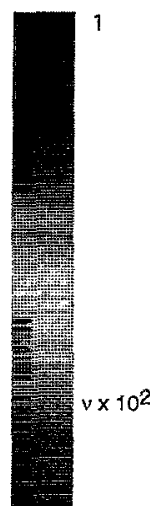

150
FIG. 4. Linear dichroism field at the boundaries between two regions covered with different numbers of molecular layers [refer to Fig. 3(b)]. The absorption anisotropy has been color coded from dark red to dark blue as the magnitude increases (see color scale). Also shown are graphs for the vertical line averages in each image of the magnitude of dichroism as a function of distance along the image. (a) and (c) represent the boundary between 31 and 21 layers, (b) and (d) represent the boundary at 21-11 layer intertace, (c) and $(\mathrm{g})$ represent the boundary at 11-5 layer interface, (f) and (b) represent the boundary between 5 and 3 layers (see text for discussion).

a region of 13 molecular layers about $60 \mu \mathrm{m}$ wide. The fact that the microscope can image the boundary between a region with 5 molecular layers and 3 molecular layers, and detect these bands shows that it can resolve differences in anisotropic absorption that correspond to the contribution of just two molecular layers and image the nonuniform build up of the LB film. These images represent the first spatially resolved measurements of optical anisotropy in such thin Langmuir-Blodgett films.

Measurement of the angle of orientation of linear dichroism at each pixel allows us to construct a similar image displaying the orientational distribution in the sample. The orientation image for the boundary between 31 and 21 molecular layers is shown in Fig. 5. The background color represents the absorption anisotropy (color scale shown in Fig. 4). In the foreground the orientation of the dichroism, and hence the mean direction of the polymer backbone, is shown by apolar vectors. The mean orientation angle lies along the transfer direction, and the whole field exhibits strong and uniform ordering. The orientation images corresponding to the $21 / 11,11 / 5$, and $5 / 3$ boundaries are very similar. In comparison to earlier macroscopic measurements

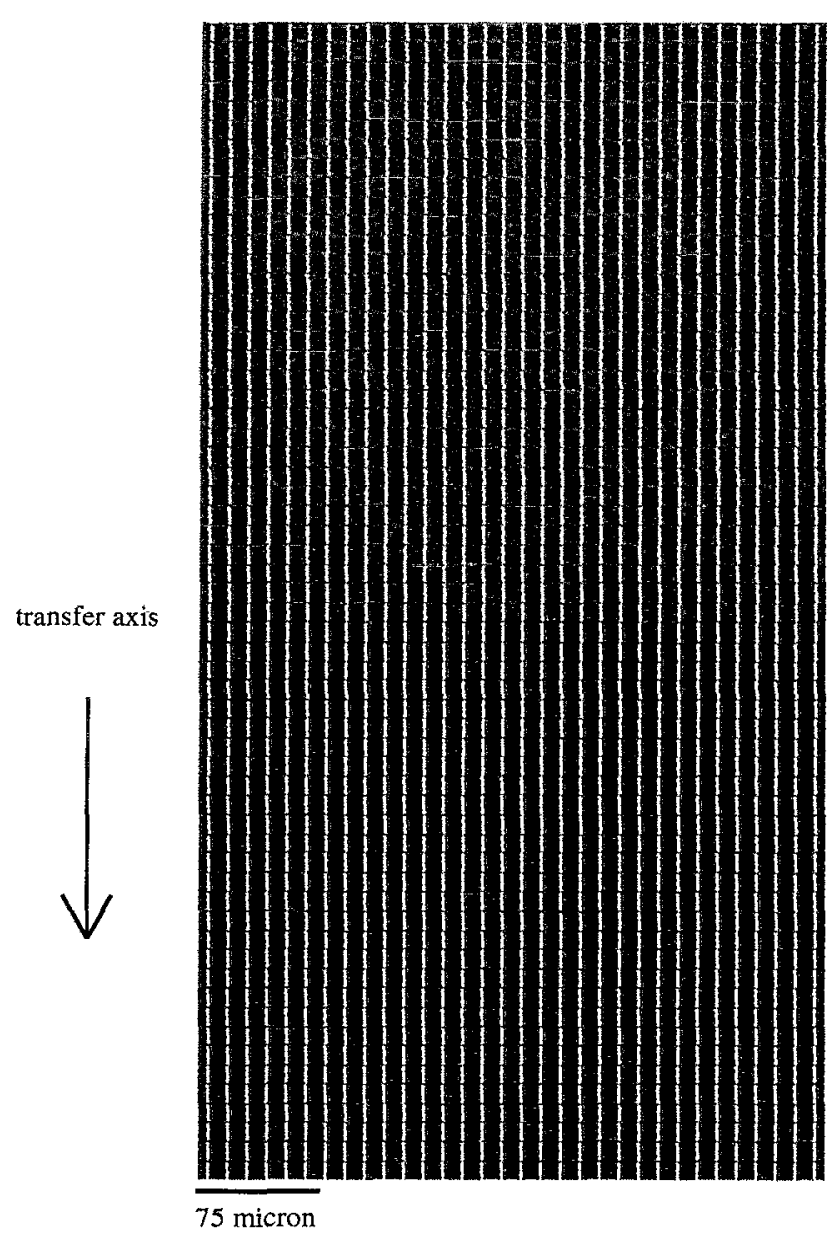

FIG. 5. The image shows the boundary between a region that is uniformly covered with 31 molecular layers of the polymer (upper half) and a region uniformly covered with 21 layers (lower half). The background shows the absorption anisotropy in a color code from dark red to dark blue as the magnitude increases. The color scale used here is shown in Fig. 4. The foreground shows the orientation angle of the dichroism, which coincides with the mean direction of alignment of the backbone of the hairy-rod polymer. The orientation image is shown on a much coarser resolution to facilitate viewing. Each vector represents the average orientation in a $8 \times 8$ pixel square, and each pixel is $1.5 \times 1.5 \mu \mathrm{m}^{2}$ (see text for discussion).

of the average orientation in LB films ${ }^{20}$ of PcPs using grazing-incidence reflection infrared (GIR-IR) spectra and polarized UV-VIS spectra, our measurements reveal that the molecular orientation is spatially uniform along the transfer direction, even on a microscopic scale. This is consistent with recent high resolution transmission electron micrographs (TEM) of LB films of PcPs and polyphthalocyaninato-germanoxane $(\mathrm{PcPg})$, that reveal a uniform mean orientation of the polymer backbone along the transfer direction on length scales of over $500 \mathrm{~nm}^{21,22}$

Rapid and sensitive characterization of molecular order by polarization-modulation laser-scanning microscopy represents a significant step in realizing the potential of LB films. Conventional methods to measure infrared or UV-VIS dichroism ${ }^{14-16,20,23}$ of such thin films require averaging over a large area and long times. Moreover, for typical LB forming compounds, observation at a molecular level under a conventional TEM causes irreversible damage by the elec- 
tron irradiation. Even for the molecules that are hardy enough to withstand the electron beam, imaging is performed under minimum dose conditions to obtain the maximum amount of structural information, and in time these films also degrade. In a broad class of biological and manufactured materials, TEM and AFM are impractical to image molecular orientational order since they can destroy the very order that is being imaged due to either irradiation or physical contact. Examination by electron microscopy places special constraints on the sample preparation procedures (e.g., LB films are either floated off glass substrates onto carbon-covered copper grids $^{21}$ or are deposited on carbon-covered nickel grids $^{22}$ ). In contrast, PM-LSM not only images orientation and order in extremely thin films in a nondestructive manner on a time scale of seconds, it does so under natural conditions, minimizing any possible artifacts.

\section{ACKNOWLEDGMENTS}

This work was supported by Raychem, and the Caltech Consortium in Chemistry and Chemical Engineering: E. I. duPont de Nemours, Eastman Kodak, and 3M. The authors thank Dr. A. Ferencz and Professor G. Wegner at the MaxPlank-Institut-für-Polymerforschung for providing the LB films. We also thank Dr. J. Revel at the Division of Biology at Caltech and Dr. P. Bauerschmidt at Zeiss for their support in modifying the Zeiss LSM, and Mr. A. Kratel for his contributions to the design of the polarization-modulating unit.

\footnotetext{
${ }^{1}$ W. Mickols, I. Tinoco, J. E. Katz, M. F. Maestre, and C. Bustamante, Rev. Sci. Instrum. 56, 2228 (1985).

${ }^{2}$ W. Mickols, M. F. Maestre, and I. Tinoco, Nature 328, 452 (1987).
}

${ }^{3}$ W. Mickols and M. F. Maestre, Rev. Sci. Instrum. 59, 867 (1988).

${ }^{4}$ G. H. Brown and J. J. Wolken, Liquid Crystals and Biological Structures (Academic, New York, 1979).

${ }^{5}$ A. Lagarde, in Optical Methods in Mechanics of Solids (Sijthoff and Noordhoff, Alphen aan den Rijn, 1981).

${ }^{6} \mathrm{C}$. J. Sheppard and T. Wilson, Theory and Practice of Optical Scanning Microscopy (Academic, New York, 1985).

${ }^{7}$ V. Wilke, Proc. SPIE 396, 164 (1984).

${ }^{8}$ V. Wilke, Scanning 7, 88 (1985).

${ }^{y}$ R. A. Azzam and N. M. Bashara, Ellipsometry and Polarized Light (North-Holland, Amsterdam, 1987).

${ }^{10}$ J. A. Schellman, Polarized Spectroscopy of Ordered Systems, edited by B. Samori and W. Thulstrup (Kluwer Academic, Dordrecht, 1987).

${ }^{11}$ G. G. Fuller, Annu. Rev. Fluid Mech. 22, 387 (1990).

${ }^{12}$ C.-B. Juang, L. Finzi, and C. J. Bustamante, Rev. Sci. Instrum. 59, 2399 (1988).

${ }^{13}$ S. J. Johnson, P. L. Frattini, and G. G. Fuller, J. Colloid Interface Sci. 104, 440 (1985).

${ }^{14}$ T. Buffeteau, B. Desbat, and J. M. Turlet, Appl. Spectrosc. 45, 1991 (1991).

${ }^{15}$ D. Blaudez, T. Buffeteau, B. Desbat, M. Orrit, and J. M. Turlet, Thin Solid Films 210, 648 (1992).

${ }^{16}$ D. Blaudez, T. Buffeteau, J. C. Cornut, B. Desbat, N. Escafre, M. Pezolet, and J. M. Turlet, Appl. Spectrosc. 47, 869 (1993).

${ }^{17}$ E. Orthmann and G. Wegner, Angew. Chem. Int. Ed. Engl. 25, 1105 (1986).

${ }^{18}$ G. Duda, A. J. Schouten, T. Arndt, G. Lieser, G. F. Schmidt, C. Bubeck, and G. Wegner, Thin Solid Films 159, 221 (1988).

${ }^{19}$ G. Duda and G. Wegner, Macromol. Chem. Rapid Commun. 9, 495 (1988).

${ }^{20}$ T. Sauer, T. Arndt, D. N. Batchelder, A. A. Kalachev, and G. Wegner, Thin Solid Films 187, 357 (1990).

${ }^{21}$ K. Yase, S. Schwiegk, G. Lieser, and G. Wegner, Thin Solid Films 210211, 22 (1992).

${ }^{22}$ F. W. Embs, E. L. Thomas, A. Gittinger, and L. Dulog, Thin Solid Films 237, 217 (1994).

${ }^{23} \mathrm{H}$. Menzel, B. Weichart, and M. L. Hallensleben, Thin Solid Films 223, 181 (1993). 